\title{
Determination of the complex stability of zinc with carbonic anhydrase in sea-water
}

\author{
Géraldine Sarthou $\dagger$, Constant M. G. van den Berg* and Ulf Riebesellł \\ Oceanography Laboratories, University of Liverpool, Liverpool, UK L69 3BX. \\ E-mail:vandenberg@liv.ac.uk; Fax:+44151794 4099
}

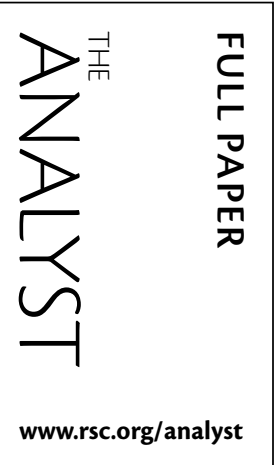

Received 19th June 2001, Accepted 20th August 2001

First published as an Advance Article on the web 10th October 2001

Carbonic anhydrase (CA) is inactive unless associated with zinc, with possible substitution by cobalt. In this work, the complexation of zinc by CA was determined in sea-water using cathodic stripping voltammetry (CSV) with ligand competition. The zinc was found to be released from the CA over a period of $3 \mathrm{~h}$ when equilibrated with a competing complexing ligand and the complex was re-formed with the CA when zinc was added. A value of 8.90 \pm 0.27 was found for $\log K_{\mathrm{ZnCA}}^{\prime}$ where $K_{\mathrm{ZnCA}}^{\prime}$ is the conditional stability constant for the complex of $\mathrm{Zn}^{2+}$ with CA in $\mathrm{pH} 8$ sea-water. A value for the molecular weight of $\mathrm{CA}$ was calculated from its equivalent ligand concentration (in $\mathrm{nM}$ ) obtained by titrations with zinc at various CA concentrations $\left(1-4 \mathrm{mg} \mathrm{1^{-1 }}\right)$. The value found $(34740 \mathrm{~g}$ $\mathrm{mol}^{-1}$ ) for the molecular weight is consistent with values found previously by other methods (29000-31000 g $\mathrm{mol}^{-1}$ ) confirming that the stoichiometry of the complex between zinc and CA is $1: 1$. This work confirms that the zinc-CA complex is reversible and that the interaction between zinc and CA can be determined using CSV with ligand competition.

\section{Introduction}

Zinc is a cofactor of several enzyme systems and it constitutes the active centre of carbonic anhydrase (CA). Carbonic anhydrase is known to be active intra- and extracellularly in microalgae from freshwater and marine environments, ${ }^{1,2}$ but it also occurs in mammals and terrestrial plants. ${ }^{3}$ Extracellular carbonic anhydrase catalyses the kinetics of carbon uptake by algae and its expression is enhanced in low $\mathrm{CO}_{2}$ conditions. ${ }^{4}$ External CA could increase the overall dissolved inorganic carbon (DIC) supply if carbon dioxide $\left(\mathrm{CO}_{2}\right)$ is the preferred species of DIC. 5,6 Thus, very low, free zinc concentrations in surface sea-water could reduce the external CA activity and then affect the uptake of inorganic carbon. It has been suggested that primary productivity is co-limited by zinc and $\mathrm{CO}_{2}^{7}$ as the growth-limiting effect of zinc is partly compensated by increasing the availability of $\mathrm{CO}_{2}$.

It has been shown that the interaction of metals with human apocarbonic anhydrase $\mathrm{B}$ is reversible, resembling metal complexes: ${ }^{8}$ zinc could be taken out of the enzyme, causing the activity to decrease, and the activity could be restored by adding cobalt which replaced the zinc. The complex stability of human CA is known to be strong, even leading to the suggestion that it could be used as a $\mathrm{Zn}^{2+}$ sensor. ${ }^{9}$ Assuming that the CA complex with zinc is reversible in sea-water (i.e., zinc can be released and thus the CA inactivated), the activity of external CA (on the cell wall of marine algae) would be affected by competition for zinc with natural complexing ligands or by competition for CA by other metals. The stability constant of the zinc-CA complex and its reversibility have not been investigated before in natural waters. Here, preliminary experiments are presented in which the interaction of zinc and CA was determined in sea-water in order to confirm whether a reversible complex is formed and whether this can be determined using voltammetry. The complex stability of $\mathrm{Zn}$ with $\mathrm{CA}$ was measured using ligand competition against pyrrolidine dithiocarbamate (PDC) with

† Present address: UMR6539/LEMAR/IUEM, F-29280, Plouzané, France.

$\$$ Present address: AWI, D-27515 Bremerhaven, Germany. detection by cathodic stripping voltammetry (CSV). ${ }^{10}$ Seawater was used to mimic conditions of external CA on marine algae. Bovine CA was used as a model for marine CA for these preliminary experiments.

\section{Experimental}

\section{Equipment}

The electrochemical equipment consisted of an Autolab voltammeter (Ecochemie) interfaced with a Metrohm (663 VA stand) hanging mercury drop electrode (HMDE). The reference electrode was double-junction, $\mathrm{Ag} / \mathrm{saturated} \mathrm{AgCl}, 3 \mathrm{M} \mathrm{KCl}$ and the salt-bridge was filled with $3 \mathrm{M} \mathrm{KCl}$; the working electrode was an HMDE and the counter electrode was a platinum wire. The solution was stirred with a Teflon-coated stirring rod; the voltammetric cell was glass.

\section{Procedures}

All plastic ware, and also the voltammetric cell and electrodes, were acid-washed with dilute, quartz-distilled hydrochloric acid before use. An aqueous solution of $0.025 \mathrm{M}$ of the ammonium salt of PDC (also called pyrrolidine- $N$-carboxydithioic acid) was prepared in water purified with a Milli-Q system (Millipore) and cleaned by three successive extractions with subboiling quartz-distilled chloroform $\left(\mathrm{QCHCl}_{3}\right)$ using aliquots of 10,5 and $5 \mathrm{ml}$. A $0.5 \mathrm{M}$ tris(hydroxymethyl)methylamine (TRIS) $\mathrm{pH}$ buffer solution (giving $\mathrm{pH} 8.07$ when added to seawater) was cleaned similarly by addition of $0.025 \mathrm{mM}$ PDC followed by three successive extractions with $\mathrm{QCHCl}_{3}$. The CA enzyme used here was dialysed and lyophilised powder from bovine erythrocytes (Sigma).

The sea-water used for these experiments originated from the North Atlantic (Discovery cruise D221, as part of the PRIME programme of the NERC) and was filtered through $0.45 \mu \mathrm{m}$ 
acid-washed polycarbonate Nuclepore filters using an in-line filtration system with a peristaltic pump.

The procedures to determine zinc concentrations and the concentration of zinc-complexing ligands were modified from van den Berg. ${ }^{10}$ Total dissolved zinc was determined after acidification to $\mathrm{pH} 2$ by addition of $10 \mu \mathrm{l}$ of $50 \%$ quartz-distilled $\mathrm{HCl}$ per $10 \mathrm{ml}$ of sample and UV irradiation for 3-4 h. The $\mathrm{pH}$ was then neutralised with distilled ammonia. A $10 \mathrm{ml}$ aliquot of the sample was pipetted into the electrochemical cell and TRIS $(5 \mathrm{mM})$ and PDC $(125 \mu \mathrm{M})$ were added. The solution was purged with nitrogen to remove oxygen, a new mercury drop was made and the $\mathrm{Zn}$-PDC complexes were adsorbed on the HMDE at a potential of $-1.3 \mathrm{~V}$ using an adsorption time of 60-120 s, whilst stirring the solution. The stirrer was stopped and the potential was set to $-0.85 \mathrm{~V}$ during a quiescence period of $10 \mathrm{~s}$. The reduction current was measured during a potential scan from -0.85 to $-1.3 \mathrm{~V}$, using square-wave modulation (50 $\mathrm{Hz}$, pulse height $25 \mathrm{mV}$, step height $2.5 \mathrm{mV}$ ). The procedure was repeated after standard additions of zinc. The detection limit of the method was $0.3 \mathrm{nM}$, determined from three times the standard deviation of $10 \mathrm{CSV}$ determinations of zinc in aliquots of Milli-Q-purified water containing $0.7 \mathrm{nM}$ of zinc.

The concentration of zinc-complexing ligands was determined by titration with zinc whilst following the labile zinc concentration by CSV with ligand competition. TRIS $(5 \mathrm{mM})$ and PDC $(25,50$ or $100 \mu \mathrm{M})$ were added to $110 \mathrm{ml}$ of sea-water. Aliquots of $10 \mathrm{ml}$ of this sea-water were pipetted into 11 polystyrene vials. Each aliquot was spiked with zinc increasing in a range between 0 and $80-150 \mathrm{nM}$ in 10 steps; the 11th vial was used as a duplicate without added zinc. The aliquots were equilibrated overnight to establish a competitive equilibrium between the added PDC, the natural organic ligands (including $\mathrm{CA}$ ) in the sample and the zinc. An adsorption potential of $-0.85 \mathrm{~V}$ was used for the determination of zinc complexation, rather than the more negative $-1.3 \mathrm{~V}$ used for total dissolved zinc, which could cause dissociation of $\mathrm{Zn}-\mathrm{CA}$ complexes during the adsorption step. ${ }^{11}$ The reduction current of the adsorbed Zn-PDC complexes was measured by CSV during a potential scan from -0.85 to $-1.3 \mathrm{~V}$.

\section{Data treatment}

The CSV data were fitted to the following equation:

[labile $\mathrm{Zn}] /[\mathrm{ZnCA}]=[$ labile $\mathrm{Zn}] / C_{\mathrm{CA}}+\alpha^{\prime} /\left(C_{\mathrm{CA}} K_{\mathrm{ZnCA}}^{\prime}\right)$

where [labile $\mathrm{Zn}$ ] is the concentration of labile zinc (determined by $\mathrm{CSV}$ ), $[\mathrm{ZnCA}]$ is the concentration of zinc bound by $\mathrm{CA}$ (calculated from total-labile zinc), $C_{\mathrm{CA}}$ is the concentration of $\mathrm{CA}$ (in $\mathrm{nM}$ of $\mathrm{Zn}^{2+}$-binding equivalents), $K_{\mathrm{ZnCA}}^{\prime}$ is the conditional stability constant for CA complexation by $\mathrm{Zn}^{2+}$ (the sidereactions of $\mathrm{CA}$ with the major cations in seaweater, most importantly $\mathrm{Ca}^{2+}$ and $\mathrm{Mg}^{2+}$, are unknown) and $\alpha^{\prime}$ is the overall side-reaction coefficient of zinc:

$$
\alpha^{\prime}=\alpha_{\mathrm{Zn}}+\alpha_{\mathrm{PDC}}
$$

where $\alpha_{\mathrm{Zn}}$ is the inorganic alpha-coefficient of zinc, which equals 2.1 at $\mathrm{pH} 8.3$ (calculated using an ion-pairing model using metal stability constants ${ }^{12}$ ), and $\alpha_{\mathrm{PDC}}$ is the alphacoefficient for zinc with PDC which was fixed by the concentration of PDC added to the solution:

$$
\alpha_{\mathrm{PDC}}=\beta_{\mathrm{ZnPDC} 2}^{\prime}\left[\mathrm{PDC}^{\prime}\right]^{2}
$$

A value of $10^{8.48}$ was used here for $\beta_{\text {ZnPDC2 }}^{\prime}{ }^{13}$

The detected CA concentration (equivalent concentration of bound zinc) and the conditional stability constant were calculated from the linear relationship between the ratios [labile $\mathrm{Zn}] /[\mathrm{ZnAC}]$ and [labile zinc] [eqn. (1)].

\section{Results}

\section{Reversibility of Zn-CA complexes}

First, a preliminary experiment was carried out to verify whether the zinc-CA complex can dissociate by determining whether zinc is released from CA after addition of PDC as a competitive ligand for zinc. In further experiments the complex formation was verified using titrations with zinc.

Filtered sea-water was UV irradiated to destroy any natural ligands originally present. A $10 \mathrm{ml}$ volume of this UV-irradiated sea-water was pipetted into the electrochemical cell and the CSV response in the presence of $125 \mu \mathrm{M}$ PDC was established. The CSV response was then followed as a function of time after addition of $2 \mathrm{mg}^{-1}$ of CA (Fig. 1). The CA addition caused an immediate decrease in the peak height, presumably due to a surfactant effect of the CA by competitive adsorption on the mercury drop. Then the peak height gradually increased with time owing to a release of zinc, the increase levelling off after $180 \mathrm{~min}$.

This result showed (1) that zinc complexed with CA is released in competition with PDC and (2) that the reaction rate of the dissociation step is slow, requiring at least $3 \mathrm{~h}$ for equilibration. Subsequently titrations with zinc were used to verify whether the complex with CA is formed in sea-water and to determine the complex stability by competitive ligand equilibration (overnight) against DPC.

\section{Titrations with zinc at various concentrations of PDC}

Complexing ligand titrations were carried out, using ligand competition against PDC, to determine the concentration of complexing sites equivalent to a given concentration of $\mathrm{CA}$ and the stability of complexes formed with zinc, in filtered UVirradiated sea-water. CA $\left(4 \mathrm{mg} \mathrm{l}^{-1}\right)$ was added to sea-water and titrated with zinc at several concentrations of PDC (25, 50 and $100 \mu \mathrm{M})$ in order to see if different sites would be detected at different detection windows. ${ }^{14}$

The CSV response (reflecting labile zinc) in the presence of $\mathrm{CA}$ as a function of the total zinc concentration was curved [Fig.2(a)], indicating that part of the added zinc was complexed by the CA in sea-water. This, in combination with the zinc release when PDC is added to CA, indicates that these complexes are chemically reversible. The variation of the detection window showed no systematic change in the detected ligand concentration (Table 1), showing that zinc was complexed at a single site. The linearisation of the data ([labile zinc]/[organically complexed zinc] as a function of the labile zinc concentration) at the three detection windows [Fig. 2(b)] gave good agreement for the ligand concentration (107 $\pm 4 \mathrm{nM}$,

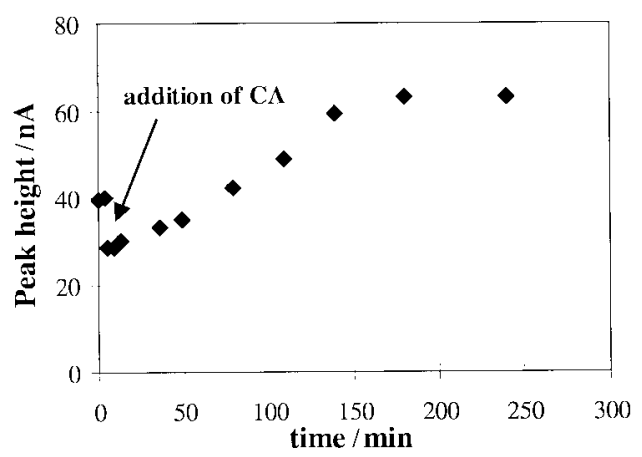

Fig. 1 CSV response for zinc (peak height/nA) as a function of time (min) before and after the addition of $2 \mathrm{mg} \mathrm{l}^{-1}$ of CA to filtered UV-irradiated seawater. The $\mathrm{CA}$ addition initially reduced the response and then the peak height increased owing to the release of zinc. 
Table 1) and the conditional stability constant $(8.54 \pm 0.09$, Table 1).

\section{Titrations at various concentrations of $\mathrm{CA}$}

Titrations were carried out at several CA concentrations to verify the ligand concentration associated with a given concentration of CA and the stability constant of its complexes with zinc. Three CA concentrations $\left(0,1,2\right.$ and $\left.4 \mathrm{mg} \mathrm{l}^{-1}\right)$ were titrated with zinc in filtered UV-irradiated sea-water. Without added CA the titration curves were straight [Fig. 3(a)], showing that any natural ligands in the sea-water had been destroyed by the UV irradiation. Titration curves after addition of 1,2 and 4 $\mathrm{mg} \mathrm{l}^{-1}$ of CA to the sea-water are shown in Fig. 3(a). The labile zinc concentration was progressively lowered at higher CA concentrations because of the competitive complexation of zinc by PDC and CA. Ligand concentrations and the conditional stability constant of $\mathrm{Zn}-\mathrm{CA}$ were calculated after linearisation of the data [Fig. 3(b)], assuming 1:1 complexation of zinc by the $\mathrm{CA}$ in sea-water, analogous to the known stoichiometry of the $\mathrm{Zn}$ complex with CA. ${ }^{15}$ The detected CA concentrations ( $\mathrm{Zn}$ complexing capacities) were found to increase linearly with the added CA concentration (Table 2). The average ligand concentration due to $\mathrm{CA}$ was $28.8 \pm 5.0 \mathrm{nmol}(\mathrm{mg} \mathrm{CA})^{-1}$. Assuming 1:1 complexation, this is equivalent to a molecular weight of $3440 \mathrm{~g} \mathrm{~mol}^{-1}$. This molecular weight is consistent with previously determined values of $29000^{16}$ and $31000^{17}$ and confirms that our assumption of $1: 1$ stoichiometry of the $\mathrm{Zn}-$ CA complex in sea-water was reasonable.
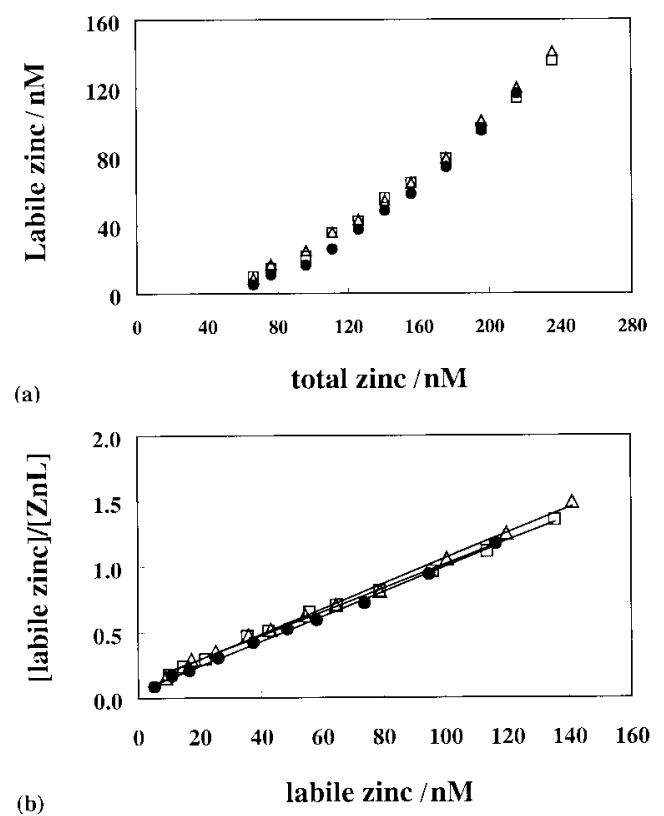

Fig. 2 Plots of the titrations of CA in filtered UV-irradiated sea-water with zinc at three detection windows. (a) Labile zinc (nM) versus total zinc (nM) and (b) [labile $\mathrm{Zn}] /[\mathrm{ZnL}]$ versus labile zinc (nM) at PDC concentrations of (๑) $25,(\triangle) 50$ and $(\square) 100 \mu \mathrm{M}$.

Table 1 CA concentrations (in $\mathrm{nM}$ equivalents of zinc bound) and conditional stability constants of $\mathrm{Zn}-\mathrm{CA}$ complexes $\left(K_{\mathrm{ZnCA}}^{\prime}\right)$ found with three different PDC concentrations $(25,50$ and $100 \mu \mathrm{M})$

\begin{tabular}{cll}
\hline PDC concentration/ $\mu \mathrm{M}$ & $C_{\mathrm{CA}} / \mathrm{nM}$ & $\log K_{\mathrm{ZnCA}}^{\prime}$ \\
\hline 25 & $106.0 \pm 1.6$ & $8.59 \pm 0.11$ \\
50 & $104.1 \pm 2.1$ & $8.43 \pm 0.09$ \\
100 & $111.2 \pm 2.3$ & $8.60 \pm 0.08$ \\
Average & $107.1 \pm 3.6$ & $8.54 \pm 0.09$ \\
\hline
\end{tabular}

\section{Discussion}

These experiments indicate that zinc complexed with $\mathrm{CA}$ is released (over a period of $3 \mathrm{~h}$ ) when equilibrated with a competing ligand and that the complex is formed when zinc is added to CA. The complex is therefore chemically reversible (albeit with slow kinetics) and CA can be treated as a normal complexing ligand for zinc. The speciation between zinc and CA can be calculated using conventional thermodynamic equations as the intervention of biological reactions is not required for the zinc-CA complex formation.

These experiments were carried out using CA from bovine origin, which has been used before as an analogue for marine extracellular algal CA. ${ }^{18}$ However, it is not certain that these findings can be extrapolated to the marine environment to predict whether the enzymatic activity of external, marine, algal $\mathrm{CA}$ is limited by the chemical speciation of zinc. Marine algal $\mathrm{CA}$, purified and concentrated, was not available for these experiments and is not commercially available. However, it is intended to extend this work to marine algal CA when such material becomes available.

The complex stability of the zinc-CA (bovine) complex in sea-water $\left(\log K_{\mathrm{ZnCA}}^{\prime}=8.9\right)$ compares with a value of 10.5 for $\log K_{\mathrm{ZnCA}}$ for human apocarbonic anhydrase $\mathrm{B}$ at $\mathrm{pH}$ 5.5. ${ }^{8}$ It is likely that in sea-water the complexation is subject to competition by the major cations which would tend to lower the apparent complex stability. The lower complex stability found in this work is therefore in line with expectation.

On the whole, this work demonstrates that CSV with ligand competition can be used successfully to determine the interaction of metals with metalloenzymes generally and specifically

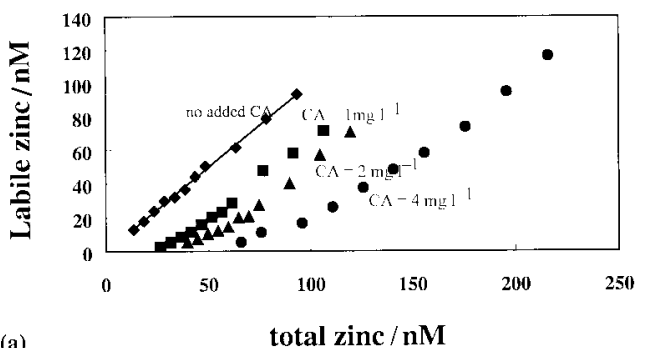

total zinc/nM

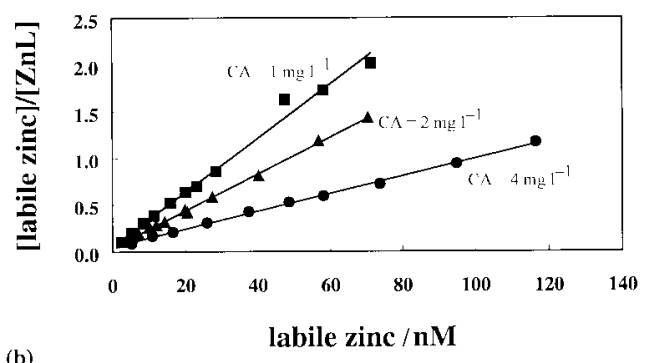

Fig. 3 Zinc titrations in the absence and presence of $\mathrm{CA}$ at various concentrations. (a) Labile zinc (nM) versus total zinc (nM) and (b) [labile $\mathrm{Zn}] /[\mathrm{ZnL}]$ versus labile zinc $(\mathrm{nM})$ at several CA concentrations: $(\boldsymbol{\nabla}) 0,(\mathbf{\square})$ $1,(\boldsymbol{A}) 2$ and (O) $4 \mathrm{mg} \mathrm{l}^{-1}$.

Table 2 Complexing ligand concentrations (based on nM zinc complexing capacities) and conditional stability constants found at three CA concentrations $\left(1,2\right.$ and $\left.4 \mathrm{mg}^{-1}\right)$

\begin{tabular}{lrr}
\hline CA concentration/mg 1-1 & \multicolumn{1}{c}{$C_{\mathrm{CA}} / \mathrm{nM}$} & \multicolumn{1}{c}{$\log K_{\mathrm{ZnCA}}^{\prime}$} \\
\hline 1 & $34.5 \pm 1.2$ & $9.08 \pm 0.37$ \\
2 & $50.8 \pm 0.6$ & $9.02 \pm 0.14$ \\
4 & $107.1 \pm 3.6$ & $8.54 \pm 0.11$ \\
Average concentration of zinc & $28.9 \pm 4.9$ & $8.88 \pm 0.29$ \\
binding sites/nmol mg & \\
\hline
\end{tabular}


of zinc with CA. Experiments are under way to determine the interaction of $\mathrm{Zn}$ with $\mathrm{CA}$ of marine algal origin, under conditions more typical of natural conditions (low $\mathrm{Zn}$ and CA).

\section{Conclusions}

This study demonstrates that CSV with ligand competition can be used to determine the interaction between metals and metalloenzymes. It is shown that bovine CA (like human CA in $\mathrm{pH}$ buffers) forms reversible complexes with zinc in sea-water: the complexes dissociate slowly over a period of $3 \mathrm{~h}$ in the presence of a competing ligand and they are formed when zinc is added to sea-water containing CA. Comparison of the molecular weight $\left(34740 \mathrm{~g} \mathrm{~mol}^{-1}\right)$ measured by the CSV titration method with previous values in the literature confirms a stoichiometry of the zinc-CA complex of $1: 1$. Experiments at various detection windows suggest that the zinc-CA complexation occurs at a single site with a specific conditional stability constant.

\section{Acknowledgements}

This study was supported by a contract from the MAS3 programme of the European Union (MAS3-CT95-0005, 'MERLIM'). The sea-water used for the experiments was collected from the North Atlantic during Discovery cruise 221 (June
1996) of the PRIME special topic (contract GST/02/1058; publication number 76) of the NERC.

\section{References}

1 A. Goyal, Y. Shiraiwa, H. D. Husic and N. E. Tolbert, Mar. Biol., 1992, 113, 349.

2 B. D. Burns and J. Beardall, J. Exp. Mar. Biol. Ecol., 1987, 107, 75 .

3 D. Hewett Emmett and R. E. Tashian, Mol. Phylogenet. Evol., 1996, 5,50 .

4 K. Palmqvist, J. W. Yu and M. R. Badger, Physiol. Plant., 1994, 90, 537.

5 S. Miyachi, M. Tsuzuki and S. T. Avramova, Plant Cell Physiol., 1983, 24, 441

6 M. Nara, Y. Shiraiwa and T. Hirokawa, Plant Cell Physiol., 1990, 31, 961.

7 F. M. M. Morel, J. R. Reinfelder, S. B. Roberts, C. P. Chamberlain, J. G. Lee and D. Yee, Nature (London), 1994, 369, 740.

8 S. Lindskog and P. O. Nyman, Biochim. Biophys. Acta, 1964, 85, 462.

9 C. C. Huang, C. A. Lesburg, L. L. Kiefer, C. A. Fierke and D. W. Christianson, Biochemistry, 1996, 35, 3439.

10 C. M. G. van den Berg, Mar. Chem., 1985, 16, 121.

11 C. M. G. van den Berg, Analyst, 1992, 117, 589.

12 D. R. Turner, M. Whitfield and A. G. Dickson, Geochim. Cosmochim. Acta, 1981, 45, 855 .

13 J. R. Donat and K. W. Bruland, Mar. Chem., 1990, 28, 301.

14 C. M. G. van den Berg and J. R. Donat, Anal. Chim. Acta, 1992, 257, 281.

15 D. Keilin and T. Mann, Nature (London), 1944, 153, 107.

16 K. P. Wong and C. Tanford, J. Biol. Chem., 1973, 248, 8518.

17 S. Lindskog, Biochim. Biophys. Acta, 1960, 39, 218.

18 D. Tchernow, M. Hassidim, B. Luz, A. Sukenik, L. Reinhold and A. Kaplan, Curr. Biol., 1997, 7, 723. 